\title{
Experiência Institucional
}

\section{Resumo}

Este texto tem como objetivo estudar e investigar a possibilidade de identificação dos traços autísticos em bebês. O cuidado precoce quanto às questões psíquicas possibilita a prevenção de psicopatologias na infância. A análise é de caráter teórico, ilustrada por recortes de casos clínicos; a fundamentação teórica é pautada por uma revisão crítica da literatura especializada, por meio de artigos cientificos e livros. Os resultados assinalam avanço científico de pesquisas que apontaram a importância e a eficácia da deteç̧ão precoce de risco psíquico, incluindo o autismo. Descritores: transtorno autístico; prevenção primária; psicanálise.

\section{REFLEXÕES ACERCA DA POSSIBILIDADE DE PREVENÇÃO DO AUTISMO}

\author{
Fernanda Delai Lucas Adurens \\ Maribél de Salles de Melo
}

DOI: http//dx.doi.org/10.11606/issn. 1981-1624.v22i1p150-165

\section{Introdução}

autismo ocupa lugar de relevância e recebe grande destaque nas discussões atualmente. Os estudos dedicados ao diagnóstico do autismo tiveram origem em Kanner, na década de 1940, com Os distúrbios do contato afetivo (1943/1997), que

- Psicóloga. Especialista em Clínica Interdisciplinar em Estimulação Precoce pela Universidade do Contestado (UnC-SC), membro do Centro Interdisciplinar de Diagnóstico e Tratamento Precoce dos Distúrbios Globais do Desenvolvimento, Espaço Escuta, Londrina, PR, Brasil.

- - Psicanalista. Analista membro da Associação Psicanalítica de Curitiba. Especialista em Clínica Interdisciplinar em Estimulação Precoce pela Universidade do Contestado (UnC-SC) e em Transtornos do Desenvolvimento Infantil e Adolescência do

Centro Lydia Coriat pelo Centro Universitário de Votuporanga (Unifev). Diretora clínica do Espaço Escuta e supervisora do Centro Mãe Paranaense (Cismepar), Londrina, PR, Brasil. 
caracterizava comportamentos de isolamento extremo, indiferença diante das pessoas e das situações, estereotipias e dificuldades severas relacionadas à linguagem, entre outros aspectos.

Atualmente, alguns autores defendem a ideia de que vivenciamos um período de epidemia relacionada ao autismo, em razão do número crescente de casos diagnosticados com esse quadro clínico. De acordo com Alfredo Jerusalinsky (2015a), nas décadas de 1970 e 1980 a contagem dos casos de autismo infantil precoce (AIP) trazia como estatística a proporção de um autista para cada 25 mil crianças. Nessa perspectiva, Laurent (2012) aponta que a nova terminologia que classifica a psicopatologia "Transtorno do Espectro Autista" ampliou o número de pessoas diagnosticadas, afirmando que, em um prazo de vinte anos, o número de crianças com esse diagnóstico multiplicou-se em dez vezes, atingindo a estatística de uma a cada 150 crianças. Diante disso, profissionais dedicados ao trabalho pautado na psicanálise com crianças têm investido em estudos direcionados à prevenção.

Pesquisas foram desenvolvidas, inicialmente na França, com o Programme de Recherche et Évaluation sur l'autisme (PREAUT), e no Brasil, com os Indicadores de Risco para o Desenvolvimento Infantil (IRDI), com o objetivo primordial de detecção precoce dos sinais de risco psíquico em bebês, visando a proporcionar o cuidado prévio. Detectados os riscos, de acordo com a gravidade dos casos se faz necessário o encaminhamento à estimulação precoce.

Diante de um cenário que traz à tona uma problemática envolvendo a saúde pública, o objetivo deste trabalho é investigar a possibilidade de identificação precoce dos traços autísticos em bebês, bem como os métodos relacionados à prevenção. Nesse intuito, seu olhar volta-se para uma estrutura psíquica ainda não decidida, tornando possível um cuidado precoce.

A pesquisa é de caráter teórico, alinhavada com recortes de clínicos. Assim sendo, encontra-se fundamentada sob a luz de conceitos psicanalíticos de autores contemporâneos dedicados aos estudos da teoria e da abordagem freudolacaniana. Os materiais utilizados foram os livros e os artigos científicos a propósito da literatura psicanalítica que abordam questões referentes ao tema proposto.

\section{Fundamentação teórica}

\section{O autismo e constituição subjetiva}

O autismo é um tema bastante estudado atualmente e delineia-se como assunto de interesse de várias especialidades. Todavia, ao mesmo tempo que conhecimentos 
a seu respeito se dilatam, as contradições diante de seu diagnóstico e das condutas a serem adotadas persistem (Quaresma \& Silva, 2011).

Kanner, em 1938 (citado por Kupfer, 2000), define o que chamou de "autismo infantil precoce" ou "distúrbio do contato afetivo" considerando-o uma síndrome específica. Tratava-se de uma população que apresentava dificuldades no campo da linguagem, nas relações interpessoais e com ruídos estridentes, mas também eram dotados de ótima memória e cognição (Kupfer, 2000).

Atualmente, existem pontos de vista e abordagens distintos que tentam explicar o autismo. Nesse intuito, a psicanálise aponta para os seguintes aspectos como relevantes nas intervenções com autismo: o psíquico, o social e o orgânico, destacando como primordiais as relações de desejo para que haja a constituição subjetiva e o surgimento do sujeito desejante. Para Sibemberg (1998), "a direção da cura do autismo em psicanálise aponta seu vetor para a constituição de um sujeito psíquico que ali falta, de modo que toda a sua aprendizagem apareça como consequência da sua inclusão subjetiva no campo significante" (p. 65).

Sabe-se que o laço com o Outro é fundamental para a constituição subjetiva. Julieta Jerusalinsky (2011) contribui com essa afirmação quando assinala que a "função materna tem um papel decisivo para a constituição subjetiva do psiquismo do recém-nascido. . . . a sua satisfação pulsional não ocorre de modo isolado ou individual, mas visa atingir, se endereça ao outro" (p. 16). Sendo assim, as fraturas nessa relação podem afetar diretamente o bebê ou a criança, fazendo surgir os traços que indicam os riscos psíquicos.

Cullere-Crespin (2010) ressalta que a psicanálise considera não somente as funções parentais como organizadoras da subjetividade do bebê, mas também uma apetência do próprio indivíduo; uma participação ativa desde seu nascimento, que o torna conectado e desejante ante o outro que lhe é referência, comumente a mãe, construindo assim sua subjetividade na relação com esse outro primordial.

Nessa direção, pesquisas atuais em psicanálise apontam que o autismo não está exclusivamente ligado a determinada fragilidade na relação entre os pais e o bebê, por motivos singulares relacionados à história desses pais. Advertem que existem casos em que os pais estão bem posicionados no exercício da função e o bebê é quem não responde à convocatória deles, resultando em um prejuízo no circuito pulsional com consequência na subjetividade, como descrito no artigo "Godente ma non troppo: o mínimo de gozo do outro necessário para a constituição do sujeito”, de Laznik (2013). 
Nesse campo de observação, a contribuição da medicina apura o olhar direcionado aos aspectos orgânicos, atentando para transtornos de neurodesenvolvimento. Muratori (2014) explica que o autismo é um transtorno neurobiológico de origem genética sem um marcador biológico, ou seja, o diagnóstico ainda se faz pela observação do comportamento. A atual versão do Manual Diagnóstico e Estatístico de Transtornos Mentais (DSM-V) (Associação Psiquiátrica Americana [APA], 2014) incluiu o autismo no campo dos Transtornos do Espectro Autista (TEA) e, com isso, integrou os diagnósticos diferenciais em função da ampla dimensão do espectro autístico, sendo assim catalogados em uma mesma categoria, cuja distinção ocorre a partir da intensidade dos sintomas e dos comportamentos (Sibemberg, 2015).

Esse fato justifica a hipótese levantada por alguns autores como Alfredo Jerusalinsky (2015a), Julieta Jerusalinsky (2015) e Laurent (2012) sobre uma possível epidemia relacionada ao diagnóstico de autismo em razão da constatação estatística do número estarrecedor de casos. De acordo com a visão de Sibemberg (2015), para a psicanálise, a anulação da diferença entre as estruturas autismo e psicose gera uma discussão teórica significativa e com consequências clínicas no que concerne às defesas de ambas as estruturas.

Entre as discordâncias de leituras para o autismo há um ponto em comum no que diz respeito a seu diagnóstico e tratamento precoce. Para as diversas visões, quanto antes houver uma intervenção, maior é a possibilidade de minimizar as dificuldades que afetam o desenvolvimento e a constituição da subjetividade da criança. É, portanto, a partir desse aspecto que pensaremos o trabalho de prevenção.

\section{Reflexões sobre a prevenção}

A noção de prevenção não pertence à psicanálise, embora se aproxime dela no que diz respeito à atividade clínica com bebês. Para alguns psicanalistas, está associada à noção de antecipação, ou seja, da precipitação do sujeito diante do Outro. Para Campanário (2008), "quando nos encontramos diante de agentes de função materna e paterna que não conseguem ver seu filho para além de uma libra de carne, podemos intervir antecipando um sujeito. Esta seria a única prevenção possível” (p. 142). 
Nesse âmbito, Laznik-Penot (1997) admite uma multifatorialidade relacionada à organização da síndrome autística, ainda que suas observações estejam iluminadas pelos registros propriamente psíquicos, pois, afirma, compreendem o único ponto no qual podemos intervir. A autora acredita que as intervenções têm como objetivo instaurar as estruturas psíquicas. Porém, as crianças com traços autísticos são encaminhadas para tratamento tardiamente, em torno de três anos, por vezes com mais idade, podendo prejudicar seu prognóstico, pois as instaurações no aparelho psíquico verificam-se desde muito cedo.

Assim, a idade apropriada para início do tratamento nos casos de autismo é anterior aos três anos, para um prognóstico mais preciso. As intervenções, quando realizadas precocemente, alcançam resultados positivos rapidamente em razão da melhor plasticidade neuronal no primeiro ano de vida da criança (Brasil, 2013).

Conforme Julieta Jerusalinsky (2002) ressalta, para que uma criança assim constituída atinja seu pleno desenvolvimento e consiga realizar as importantes aquisições instrumentais, não basta somente esperar a passagem do tempo cronológico e o alcance da maturação, mas, noutro sentido, urge a intervenção, preferencialmente em tempo precoce.

Com a importante descoberta de Kandel (citado por Catão, 2009) sobre o conceito de neuroplasticidade, os critérios psicopatológicos se modificam radicalmente, especialmente considerando os primeiros tempos do desenvolvimento - ou seja, os primeiros anos da criança têm função significativa na constituição da vida psíquica, e existe uma mobilidade nas formações psicopatológicas até a puberdade, tornando-as não decididas até esse momento da adolescência.

A neuroplasticidade é uma característica peculiar do sistema nervoso central e cujo mecanismo dispõe de compensação dos efeitos de lesões neuromaturacionais, ou seja, a capacidade do sistema nervoso de modificar sua estrutura a partir de padrões de experiência (Sibemberg, 1998).

Outro conceito fundamental pelo qual psicanalistas se orientam e apropriam é a noção da epigenética. Esse conceito oferece suporte teórico para a investigação da possibilidade de modificação das estruturas neuronais a partir do ambiente, favorecendo um novo olhar quanto às questões etiológicas. Trata-se de um modelo que considera a ligação entre as questões de ordem genética e os fatores ambientais, considerando as alterações intracelulares no material genético do organismo (Freitas-Silva \& Ortega, 2014).

O processo dos mecanismos epigenéticos está relacionado primordialmente à noção de que a determinação biológica do organismo pode modificar-se ao longo de seu desenvolvimento, rompendo com a ideia de um determinismo inalterável (Freitas-Silva \& Ortega, 2014). 
Esse conceito de epigenética abre margem e esperança para a parcela de psicanalistas que se dedica ao trabalho com bebês e pequenas crianças e cujo objetivo é o de cuidar e detectar os traços iniciais de autismo, ou seja, promove um olhar precoce às dificuldades apresentadas nos primeiros tempos da constituição psíquica e do desenvolvimento infantil.

Diante disso, uma equipe composta por médicos pediatras e psicanalistas desenvolveu uma pesquisa na França chamada Programme de Recherche et Évaluation sur l'autisme (PREAUT) para detectar sinais de risco indicativos de autismo com o objetivo de priorizar a precocidade do tratamento, no qual o profissional se atenta à ausência de certos sinais determinantes para a constituição do bebê com o desenvolvimento normal. Afinal, quanto mais cedo ocorre a intervenção, maior a probabilidade de um bom prognóstico.

O projeto PREAUT surgiu no ano de 1998, a princípio na França, e foi posteriormente difundido entre vários países, entre eles o Brasil. Todo o processo começa a partir da hipótese de Serge Lebovici acerca da possibilidade de alteração da constituição subjetiva de uma criança com traços autísticos caso ocorresse uma intervenção antes de o bebê completar um ano de idade (Cullere-Crespin \& Parlato-Oliveira, 2015).

A psicanalista Marie-Chistine Laznik, pouco antes do surgimento do PREAUT, debruçou-se sobre vídeos 
caseiros de bebês que posteriormente receberam diagnóstico de autismo. ${ }^{1}$ Foi então que Laznik-Penot (1997) desenvolveu sua hipótese, definindo dois pontos fundamentais envolvendo sinais de risco para o autismo: o não olhar entre o bebê e sua mãe, especialmente quando a mãe não tem noção do fato, e o fracasso do circuito pulsional completo.

De acordo com Laznik-Penot (1997), o não olhar promove uma fratura significativa no estágio do espelho, descrito por Lacan como o tempo particular do reconhecimento do bebê pelo Outro a partir da imagem especular, proporcionando ao pequeno sua imagem corporal. Acrescenta, ainda, "o que chamo aqui olhar, é também o que permite à mãe escutar de início nos balbucios do bebê, mensagens significantes que ele fará suas mais tarde. Ver e escutar o que ainda não está para que um dia possa advir" (Laznik-Penot, 1997, p. 39).

Quanto aos tempos do circuito pulsional, o primeiro tempo refere-se à busca ativa do bebê pelo objeto oral como o seio, a mamadeira. O segundo é marcado pelo autoerotismo do bebê ao se interessar em chupar seu dedo ou a chupeta, e por último, já no terceiro tempo, o bebê se lança a um Outro primordial, ou seja, estende seu pé, sua barriga para que a mãe o "morda", o "coma", aproximando mãe e bebê numa relação de prazer e gozo em que o bebê se faz objeto de um Outro. Neste último tempo, 
percebe-se uma passividade aparente da pequena criança, visto que é ativamente que se faz devorar pelo Outro.

Somente com a conclusão do terceiro tempo é possível considerar uma satisfação pulsional e o surgimento de um sujeito (Laznik-Penot, 1997). Para termos a certeza de que o bebê está engatado no circuito pulsional e que os dois primeiros tempos são, real e verdadeiramente, fruto de uma satisfação, é preciso constatar o terceiro tempo, no qual, de fato, ocorre uma troca de prazer entre mãe e bebê. Lacan (1964/1990) afirma a respeito: "no momento em que o fecho se fechou, quando é de um polo ao outro que houve reversão, quando o outro entrou em jogo, que o sujeito tomou-se por termo terminal da pulsão" (p. 173) e acrescenta que a ideia de pulsão só pode existir através da dimensão do Outro.

Sendo desse modo, a equipe partiu desses dois sinais para iniciar o projeto; porém, mostrava-se necessário um instrumento de avaliação. Para tanto, fora selecionada uma amostra de 4.822 crianças entre os anos de 2006 e 2011 e 11.808 inclusões ao final do protocolo em 2011, contando com 600 pediatras que realizaram formação específica. Nos bebês dos 4 aos 9 meses testou-se a hipótese de Laznik (2013) sobre o fechamento do circuito pulsional; aos 12 meses foi aplicado o questionário de desenvolvimento da comunicação (QDC-C); e aos 24 meses foi realizado o Checklist for Autism in toddlers (CHAT) em uma versão francesa adaptada (Cullere-Crespin \& Parlato-Oliveira, 2015).

Os resultados intermediários foram publicados em 2011. O total de crianças incluídas na pesquisa foi de 11.318; no entanto, apenas 3.765 delas concluíram as quatro etapas do protocolo. Desse total, 28 tiveram resultados positivos, ou seja, apresentaram sinais de risco, enquanto apenas 24 puderam ser analisadas, sendo que 4 foram perdidas no decorrer da pesquisa. Entre as 24, somente 2 foram diagnosticadas com transtorno autístico e 4 receberam diagnóstico de Transtorno Invasivo do Desenvolvimento (TID), ou seja, essas 4 crianças apresentavam prejuízos relacionados à linguagem, à comunicação e à interação social; e as outras 18 crianças apresentaram dificuldades e diagnósticos distintos, como atraso mental leve, déficit de atenção e hiperatividade, prematuridade e doença orgânica, entre outros.

No Brasil, essa pesquisa foi iniciada em 2007 com o apoio do Instituto da Família Viva Infância e, em 2009, recebeu o apoio do Instituto Langage. O projeto foi aprovado pelo Comitê de Ética em Pesquisa Nacional e está sendo coordenado por Erika 
Parlato-Oliveira e por Luiza Bradley de Araújo e dirige-se aos profissionais das áreas da saúde e da educação.

Também no Brasil, desenvolveu-se outra pesquisa de avaliação com o olhar voltado à prevenção: os Indicadores Clínicos de Risco do Desenvolvimento Infantil (IRDI). Trata-se de um estudo iniciado entre os anos 2000 e 2009, desenvolvido sob viés psicanalítico a partir de Freud, apoiado nas leituras de Lacan e Winnicott, sob as quais foi construído um instrumento composto por 31 indicadores clínicos de riscos psíquicos e do desenvolvimento infantil observados nos primeiros 18 meses de vida a partir do vínculo do bebê com aquele que exerce a função materna e que indica a paterna. O estudo apresenta a divisão em quatro da faixa etária de 0 a 18 meses (0-4 meses; 4-8 meses; 8-12 meses; 12-18 meses) (Brasil, 2013; Jerusalinsky, A., 2015b; Kupfer et al., 2009).

A amostra alcançada foi de 727 crianças testadas depois dos três anos de idade em 11 hospitais e centros de atenção pública em 10 capitais brasileiras. Do total de crianças, 287 apresentaram dois ou mais indicadores, e o restante se caracterizou como grupo controle. Dessas 287, sorteou-se 183 (das quais somente 158 concluíram o estudo) para construir uma subamostra para retestagem por meio da Avaliação Psicanalítica para crianças de três anos (AP3) e com a entrevista psiquiátrica "com finalidade de verificar a correlação entre os casos detectados como risco durante os primeiros 18 meses e a continuidade ou não da condição de risco aos três anos de idade" (Jerusalinsky, A., 2015b, p. 422).

Desse total de 727 crianças, 440 apresentaram somente um ou nenhum indicador ausente, sendo consideradas pelos pesquisadores, portanto, como crianças controle. Dessas 440, foram sorteadas 132 crianças para finalizarem o estudo, sendo que 122 compareceram; todavia as perdas não foram relevantes no que concerne à análise estatística dos casos (Jerusalinsky, A., 2015b). Com a validação do IRDI, o instrumento torna-se um material disponível a pediatras em consultas regulares, bem como a pesquisadores e profissionais que trabalham com bebês e, acima de tudo, a favor da saúde pública (Kupfer et al., 2009; Pesaro, 2011).

Desde seu início, a pesquisa prima por respeitar duas condições: a de detectar o risco e não realizar o diagnóstico, e a de buscar traços de saúde e não de doença, pois aí delineia-se um grande problema relacionado aos checklists que associam comportamentos diversos a patologias, gerando falsas epidemias (Jerusalinsky, A., 2015b).

Esse estudo reuniu pesquisadores de diferentes especialidades, como pediatras, psiquiatras e psicólogos. Após seu início, o Ministério da Saúde acreditou ser uma valiosa informação para a saúde pública e, junto do Conselho Nacional de Desenvolvimento Científico e Tecnológico (CNPq), da Fundação de 
Amparo à Pesquisa do Estado de São Paulo (Fapesp) e da Universidade de São Paulo (USP), apoiou a iniciativa. Sendo assim, foi possível reunir 250 pesquisadores brasileiros, incluindo metodólogos. Para a construção dos indicadores, foram estabelecidos quatro eixos teóricos: supor um sujeito, estabelecer a demanda, alternância presença-ausência, e função paterna.

Primeiramente, supor um sujeito está relacionado à importância daquele que exerce a função materna de atribuir sentidos aos objetos, aos sentimentos e às sensações, atribuindo significação ao que o bebê faz e sente para que posteriormente essa suposição venha a se tornar um diálogo, permitindo a ele apropriar-se dessas significações (Jerusalinsky, A., 2015b).

Em relação ao estabelecimento da demanda, indica o reconhecimento materno aos choros e às manifestações do bebê como ações e pedidos voltados à mãe, o que em psicanálise é nomeado de "experiência de satisfação primária”. A partir desse reconhecimento ao pedido do bebê é que se constrói uma demanda que servirá de base para a constituição da linguagem e da relação com os outros (Jerusalinsky, A., 2015b; Kupfer et al., 2009).

Quanto à alternância presença-ausência, trata-se da alternância da mãe ou de quem exerce essa função nos cuidados com o bebê. Quando a mãe se presentifica de maneira constante, evitando que o bebê experimente a falta e, assim, tenha de lidar com uma dificuldade, impõe a 
ele um impedimento para conquistar e construir sua própria identidade. Com importância, ressalta-se, aqui, que é justamente nesse primeiro tempo de vida que a criança representa, com a ausência materna, toda a ausência com o qual irá se deparar ao longo da vida. Portanto, são nesses intervalos entre presença e ausência que a pequena criança tem a oportunidade de simbolizar essa presença física (Jerusalinsky, A., 2015b; Kupfer et al., 2009).

Por fim, a função paterna diz respeito a uma terceira instância que atravessa a relação mãe-bebê, ou seja, essa função tem como objetivo permear a relação mãe-bebê fazendo referência à cultura; para tanto, a mãe precisa ter a função paterna inscrita em seu psiquismo, promovendo uma separação simbólica e, com isso, evitar que o filho ocupe um lugar de objeto somente para sua satisfação (Kupfer et al., 2009).

Esses quatro princípios psicanalíticos serviram como base aos pesquisadores na identificação dos indicadores de risco. A intenção inicial dessa pesquisa era identificar primordialmente os riscos psíquicos de psicopatologias graves como autismo e psicose; porém, a indicação principal do instrumento foi para identificação precoce de problemas no desenvolvimento, salvo alguns indicadores específicos que prediziam risco psíquico.

O estudo de Pesaro (2011), em que se propõe investigar os alcances e os limites da pesquisa IRDI, aponta a importância de se dissolver a noção de que a psicanálise não dialoga com a ciência, ressaltando que os limites dessa pesquisa estão relacionados à utilização do instrumento na saúde pública, como a incerteza dessa utilização, a forma como ele será empregado e se haverá formação dos profissionais que farão uso desse material.

Lerner (2011 citado por Mota et al., 2015, p. 466) estudou a possibilidade de o instrumento IRDI discriminar o autismo, retardo mental e normalidade. Constatou que alguns itens apontam, estatisticamente, capacidade para essa discriminação e sugere pesquisas que envolvam o instrumento IRDI para a detecção de quadros de transtorno do espectro autista. Em convergência, Campana (2013) conclui em sua pesquisa que o mesmo instrumento é capaz de detectar os casos de bebês que se encaminham para o autismo, embora reforce que o instrumento não seja específico para a detecção de traços autísticos, e sim de manifestações sintomáticas amplas que apontam risco psíquico e do desenvolvimento. Nessa direção, Machado, Lerner, Novaes, Palladino e Cunha (2014) verificaram que existe "sensibilidade" no IRDI - questionário para indicar transtornos autísticos -, recomendando, no entanto, a utilização de mais de um instrumento para a segurança e melhor assertividade nos encaminhamentos.

Detectados os primeiros sinais de risco que possam acarretar possíveis prejuízos para o bebê, é indicado o tratamento em estimulação precoce. 


\section{Estimulação Precoce como meio de prevenção}

A Estimulação Precoce (EP) ocorre a partir da intervenção na relação mãe-bebê, objetivando o suporte da função materna para se estabelecer as operações constituintes da subjetividade. Logo, não se trata de um estímulo destinado puramente ao corpo; é preciso considerar a apropriação e a inclusão do bebê no processo. Para Coriat e Jerusalinsky (1997), trabalhar com crianças não é o mesmo que trabalhar com coisas, portanto não se trata de reformar seu sistema nervoso, tampouco de organizar as informações em cada lugar específico; trata-se, antes, de sustentar ou de construir com o bebê seu lugar enquanto sujeito.

O clínico em EP precisa saber olhar para o bebê, escutar os pais e entender em que lugar simbólico o bebê encontra-se situado no desejo e no discurso parental; considerar como estão as questões fantasmáticas e reais que se apresentam, promovendo uma "ponte", a partir da transferência, entre os pais e o bebê.

Muratori (2014) afirma que a intervenção precoce antes dos três anos nos casos de autismo é capaz de dificultar os comprometimentos relacionados aos aspectos neurobiológicos, como déficits sociais e na comunicação.

No Espaço Escuta e no Centro Mãe Paranaense, instituições da cidade de Londrina, no Paraná, são atendidas gestantes e aproximadamente 300 bebês e crianças; para isso, contam com uma equipe interdisciplinar. Os encaminhamentos ocorrem cada vez mais cedo em função do trabalho de formação e de capacitação destinado aos profissionais da área da saúde e da educação da região. Os bebês iniciam o trabalho antes de um mês de vida, o que favorece o prognóstico.

Os breves recortes de casos clínicos citados a seguir são de bebês atendidos nessas instituições. O primeiro bebê chega para tratamento com três meses, sendo irmã de gemelares que vieram para tratamento aos cinco anos de idade. Apresenta recusa no olhar, não responde às brincadeiras corporais e a mãe demonstrava muita dificuldade em aceitar que mais um filho precisaria de tratamento, não conseguindo levá-la à instituição com a frequência necessária. Aos sete meses da filha, começa a perceber a necessidade do trabalho, e, nessa época, era visível sinais de risco significativos, com movimentos motores repetitivos importantes, 
falta de expressão, permanência da posição sentada, sem conseguir se deslocar, e a não exploração dos brinquedos. Pouco a pouco, por meio do tratamento em EP, foi possível reorganizar as funções. Atualmente com dois anos, apresenta intenção comunicativa, começa a organizar um brincar e já convoca a mãe e a analista nessa aventura.

O outro caso trata-se de dois bebês gemelares que também têm uma irmã mais velha que veio para tratamento aos dois anos e oito meses com diagnóstico médico de autismo. As gemelares estavam com sete meses quando foram avaliadas e apresentavam recusa no olhar, nenhuma sustentação de tronco - não sentando, portanto, sem apoio -, não respondiam a brincadeiras corporais, não balbuciavam e nem emitiam sons, tampouco expressão facial. Iniciaram tratamento em EP e, atualmente, com cinco anos, já receberam alta da instituição com o desenvolvimento em convergência com a idade.

A partir desses casos, destaca-se a importância de um olhar para os irmãos de crianças que já apresentam o diagnóstico de autismo.

A intervenção a tempo, em momento de plena constituição e desenvolvimento, proporcionou uma evolução importante do funcionamento global do bebê.

\section{Considerações finais}

Ao longo deste estudo, verificou-se a complexidade do diagnóstico de autismo, suas nuances e particularidades. As pesquisas realizadas com o objetivo de identificação precoce dos riscos psíquicos mostram um significativo avanço do ponto de vista científico. A prevenção dos traços de autismo em bebês demonstra um progresso significativo e ambicioso para saúde pública.

Outra questão importante a ser considerada relaciona-se aos encaminhamentos dos casos de risco psíquico, em razão da carência de instituições especializadas no atendimento a bebês e, especialmente, que trabalhem com viés interdisciplinar. Essa dificuldade faz que muitos casos cheguem às instituições tardiamente, apresentando os sintomas importantes já instaurados, o que pode prejudicar a evolução e o prognóstico da criança. Torna-se necessário investimento 
por parte do poder público para o tratamento desses bebês, caso contrário a eficácia da detecção dos indicadores de risco perde sua potência.

Observa-se que a psicanálise tem um papel fundamental e de relevância nos avanços das pesquisas e no tratamento do autismo. Seu diálogo com a ciência facilita a comunicação entre pesquisadores e estudiosos, comprometendo-se com o rigor científico.

\title{
REFLECTIONS ABOUT THE POSSIBILITY OF AUTISM PREVENTION
}

\begin{abstract}
This paper aims to study and investigate the possibility of identifying autistic traits in babies. Early care for mental issues enables the prevention of psychopathology in childhood. This study is a theoretical analysis illustrated by examples of a clinical case; the theoretical underpinning is guided by a critical literature review in psychoanalytics, through scientific articles and books. The results indicate scientific advancement of research pointing to the importance and effectiveness of early detection of psychological risk, including autism.
\end{abstract}

Index terms: autistic disorder; primary prevention; psychoanalysis.

\section{REFLEXIONES SOBRE LA POSIBILIDAD DE PREVENIR EL AUSTIMO}

\section{RESUMEN}

Este articulo tiene como objetivo investigar las posibilidades de identificación de los rasgos autísticos en bebés. Tener en cuenta las cuestiones psíquicas posibilita la prevención de psicopatologías en la infancia. El análisis es de carácter teórico y también se basa en el estudio de un caso clínico; el marco teórico viene determinado por una revisión crítica de la literatura especializada en psicoanálisis-articulos cientificos y libros. Los resultados señalan un gran avance en las investigaciones cientificas que apuntan la importancia y la eficacia de la detección precoz del riesgo psicológico, incluyendo el autismo.

Palabras clave: trastorno autístico; prevención primaria; psicoanálisis.

\section{REFERÊNCIAS}

Associação Psiquiátrica Americana (APA). (2014). Manual diagnóstico e estatístico de transtornos mentais (5a ed.). Porto Alegre, RS: Artes Médicas.

Brasil. Ministério da Saúde. Secretaria de Atenção à Saúde. Departamento de Ações Programáticas Estratégicas. (2013). Diretrizes de atenção à reabilitação da pessoa com transtorno do espectro do autismo. Brasília, DF: Ministério da Saúde.

Campana, N. T. C. (2013). Uso de indicadores clínicos de risco para o desenvolvimento infantil (IRDI) na deteç̧ão de sinais de problemas de desenvolvimento associados ao autismo. Dissertaçáo de Mestrado, Instituto de Psicologia, Universidade de São Paulo, São Paulo, SP.

Campanário, I. S. (2008). Espelho, espelho meu: a psicanálise e o tratamento precoce do autismo e outras psicopatologias graves. Salvador, BA: Ágalma. 
Catão, I. (2009). O bebê nasce pela boca: voz, sujeito e clínica do autismo. São Paulo, SP: Instituto Langage.

Coriat, L., \& Jerusalinsky, A. (1997). Definição de estimulação precoce. In Centro Lydia Coriat (Org.), Escritos da criança no 1 (pp. 72-75). Porto Alegre, RS: Centro Lydia Coriat.

Cullere-Crespin, G. (2010). Discussão da evolução de uma síndrome autística tratada em termos de estruturação psíquica e de acesso à complexidade. Psicologia Argumento, 28(61), 159-166.

Cullere-Crespin, G., \& Parlato-Oliveira, E. (2015). Projeto PREAUT. In A. Jerusalinsky (Org.), Dossiê autismo (A. R. N. Jerusalinsky, E. Parlato-Oliveira, P. Glei, \& R. E. O. G. Kelly, trads., pp. 436-455). São Paulo, SP: Instituto Langage.

Freitas-Silva, L. R., \& Ortega, F. J. G. (2014). A epigenética como uma nova hipótese etiológica no campo psiquiátrico contemporâneo. Physis online, 24(3), 765-786. doi: http://dx.doi. org/10.1590/S0103-73312014000300006

Jerusalinsky, A. (2015a). Dar a palavra ao autista. In A. Jerusalinsky (Org.), Dossiê autismo (A. R. N. Jerusalinsky, E. Parlato-Oliveira, P. Glei, \& R. E. O. G. Kelly, trads., pp. 12-17). São Paulo, SP: Instituto Langage.

Jerusalinsky, A. (2015b). Indicadores de Risco: como a psicanalise pode proteger os bebês. In A. Jerusalinsky (Org.), Dossiê autismo (A. R. N. Jerusalinsky, E. Parlato-Oliveira, P. Glei, \& R. E. O. G. Kelly, trads., pp. 416-433). São Paulo, SP: Instituto Langage.

Jerusalinsky, J. (2002). Enquanto o futuro não vem: a psicanálise na clinica interdisciplinar com bebês. Salvador, BA: Ágalma.

Jerusalinsky, J. (2011). A criação da criança: brincar, gozo e fala entre a mãe e o bebê. Salvador, BA: Ágalma.

Jerusalinsky, J. (2015, 30 de março). Para onde vamos com o autismo? Estadão, Sáo Paulo, Criança em Desenvolvimento. Recuperado de: http://bit.ly/2peIvRP

Kanner, L. (1997). Os distúrbios do contato afetivo. In P. S. Rocha (Org.), Autismos (pp. 111-170). São Paulo: Escuta. (Trabalho original publicado em 1943)

Kupfer, M. C. M. (2000). Notas sobre o diagnóstico diferencial da psicose e do autismo na infância. Psicologia USP, 11(1), 85-105. doi: http://dx.doi.org/10.1590/S0103-65642000000100006

Kupfer, M. C. M., Jerusalinsky, A. N., Bernardino, L. M. F., Wanderley, D., Rocha, P. S. B., Molina, S. E., . . Lerner, R. (2009). Valor preditivo de indicadores clínicos de risco para o desenvolvimento infantil: um estudo a partir da teoria psicanalítica. Latin-American Journal of Fundamental Psychopathology, 6(1), 48-68. Recuperado de: http://bit.ly/2oJlS6o

Lacan, J. (1990). O seminário, livro 11: os quatro conceitos fundamentais da psicanálise. (M. D. Magno, trad.). Rio de Janeiro, RJ: Jorge Zahar. (Trabalho original publicado em 1964)

Laurent, E. (2012). O que nos ensinam os autistas. In A. Murta, A. Calmon, \& M. Rosa (Orgs.), Autismo(s) e atualidade: uma leitura lacaniana (pp. 17-43). Belo Horizonte, MG: Scriptum Livros.

Laznik-Penot, M. C. (1997). Poderíamos pensar numa prevenção da Síndrome Autística? In D. Wanderley (Org.), Palavras em torno do berço: intervençöes precoces bebê e família (pp. 35-51). Salvador, BA: Agálma.

Laznik, M. C. (2013). Godente ma non troppo: o mínimo de gozo do outro necessário para a constituição do sujeito. In E. Parlato-Oliveira (Org.), A hora e a vez do bebê (pp. 16-35). São Paulo, SP: Instituto Langage.

Machado, F. P., Lerner, R., Novaes, B. C. A. C., Palladino, R. R. R., \& Cunha, M. C. (2014). Questionário de indicadores clínicos de risco para o desenvolvimento infantil: avaliação da sensibilidade para transtornos do espectro do autismo. Audiology Communication Research, 19(4), 345-351. doi: http://dx.doi.org/10.1590/S2317-64312014000300001392 
Mota, A. D. P., Lerner, R., Escobar, A. M. U., Kupfer, M. C. M., Rocha, F. M. M., $\&$ Santos, L. S. (2015). Associação entre sinais de sofrimento psíquico até dezoito meses e rebaixamento da qualidade de vida aos seis anos de idade. Psicologia USP, 26(3), 464-473. doi: http://dx.doi.org/10.1590/0103-656420130043

Muratori, F. (2014). Manual precoce no autismo: guia prático para pediatras (C. C. Siervi, \& D. B. Wanderley, trads.). Salvador, BA: Núcleo Interdisciplinar de Intervenção Precoce da Bahia.

Pesaro, M. E. (2011). Alcance e limites teórico-metodológicos da pesquisa multicêntrica de indicadores clínicos de risco para o desenvolvimento infantil. Associação Psicanalitica de Curitiba, 22, 145-169.

Quaresma, H. D. V., \& Silva, V. G. (2011). Autismo infantil: concepçóes e práticas psicológicas. Revista Brasileira de Ciências da Saúde, 14(4), 85-90. doi: 10.4034/ RBCS/2010.14.04.11

Sibemberg, N. (1998). Autismo e linguagem. In Centro Lydia Coriat (Org.), Escritos da criança $n^{\circ} 5$ (pp. 60-71). Porto Alegre, RS: Centro Lydia Coriat.

Sibemberg, N. (2015). Atenção com o diagnóstico: a suspeita de autismo nem sempre se confirma. In A. Jerusalinsky (Org.). Dossiê autismo (A. R. N. Jerusalinsky, E. Parlato-Oliveira, P. Glei, \& R. E. O. G. Kelly, trads., pp. 96-105). São Paulo, SP: Instituto Langage.

\section{NOTA}

1. Os vídeos foram disponibilizados pela Fundação Stella Maris, na Universidade de Pisa, devido a contatos de Laznik com dois professores desta instituiçáo (Cullere-Crespin \& Parlato-Oliveira, 2015).

fernandadla10@gmail.com

Rua Amador Bueno, 388 86010-620 - Londrina - PR - Brasil.

maribelmelo@hotmail.com

Rua Amador Bueno, 388 86010-620 - Londrina - PR - Brasil. 